\title{
5 Das Problem der Voraussetzungen der Logik
}

Trotzdem sind wir an diesem Punkt noch immer nicht über die bloße Setzung der Resultate und Voraussetzungen der Logik hinaus. Ankommen aber wird es vor allem darauf, wie diese Voraussetzungen von der Logik in Bezug auf den kategorialen Rahmen von „Realität überhaupt“ eingeholt, d.h. in der Entwicklung der Gedankenbestimmungen begründet, werden. Wer die Wirklichkeit des Begriffs aufzeigen will, muss diese in Form der logischen Methode immer schon voraussetzen: Das sieht nur dann nach einem ungebührlichen logischen Zirkel aus, wenn man die retrograde Bewegung des Einholens dieser Voraussetzungen im Voranschreiten der rein immanenten Gedankenentwicklung der Logik nicht als Schaffung qualitativ neuer Einsichten versteht, welche unabhängig von ihren Voraussetzungen so Geltung erlangen, dass sie eine echte begründende Kraft für ihre Prämissen erhalten können. Die Hegel-Forschung hat seit Trendelenburgs Kritik an den angeblich versteckten, unbegründeten Voraussetzungen gerade der $\operatorname{Logik}^{17}$ immer wieder darüber gestritten, ob es bei Hegel nicht doch letzte bzw. erste absolute Prinzipien und Grundsätze gebe, die eben nicht im System erst begründet werden, sondern diesem quasi versteckt untergeschoben sind und vielmehr alle Begründungsleistungen tragen ${ }^{18}$. Beispielsweise meint Vittorio Hösle: „Gerade eine Philosophie [wie die Hegels, C.W.], die auf dem Grundprinzip der Unhintergehbarkeit des Denkens basiert, kann nicht umhin, die Erkennbarkeit alles Seienden für nicht konsistent hinterfragbar zu halten. “19 Was aber nicht konsistent hinterfragbar ist, kann auch nicht mehr vollständig begründet werden, denn eben das heißt ja begründen: es im Hinterfragen hinreichend auf ein Anderes zurückführen. Festgehalten werden muss, dass die Logik von dem klar formulierten Programm getragen ist, die am Anfang der Logik im Begriff der Einheit ihrer Methode und des Inhalts gemachte Voraussetzung im Gang der Logik selbst explizieren und begründend einholen zu wollen. Auch der Bezug auf die vermeintlich absolute Voraussetzung des Gedankengangs der Phänomenologie

17 Vgl. Adolf Trendelenburg: Logische Untersuchungen I (1840), Reprint, Hildesheim 1964, S. 38. 18 Auch Michael Quante geht von „Grundprinzipien“ der hegelschen Philosophie aus, ohne allerdings zu behaupten, diese seien unbegründbar oder verborgen (Quante: Die Wirklichkeit des Geistes, S. 24); vgl. dazu die Rezensionen von Anton Friedrich Koch (in: Zeitschrift für Philosophische Forschung 67 (2013), S. 319-322), der gerade diese Annahme bemängelt, und Claudia Wirsing (in: Hegel-Studien 47 (2013), S. 212-216). Demgegenüber bestehen diejenigen, welche Hegel beim Wort nehmen, darauf, dass Hegels Philosophie sich gerade dadurch auszeichne, keine ersten oder letzten singulären Prinzipien zu haben, sondern alle Normen und Gehalte rein aus der Denkbewegung des „Denkens des Denkens“ zu erzeugen.

19 Hösle: Hegels System, S. 71.

Ә OpenAccess. (C) 2021 Claudia Wirsing, publiziert von De Gruyter. (cc) BY unter einer Creative Commons Namensnennung 4.0 International Lizenz. 
des Geistes ist irreführend, wo er einfach und ausschließend im Sinne eines bloßen Entweder-Oder gemeint ist. Hegel verweist in der „Einleitung“ selbst auf diese Bedingung: „Der Begriff der reinen Wissenschaft und seine Deduction wird in gegenwärtiger Abhandlung also insofern vorausgesetzt, als die Phänomenologie des Geistes nichts anderes als die Deduction desselben ist.“ (GW 21, S. 33) Gemeint ist mit dieser „reinen Wissenschaft“ das am Ende der Phänomenologie erreichte „absolute Wissen“, in welchem „die Befreyung von dem Gegensatze des Bewußtseyns“ (GW 21, S. 33) zwischen Denken und Welt, Selbstbeziehung des Ich und gegenständlicher Objektivität erreicht wurde. Folglich muss einerseits festgehalten werden: Das „reine Wissen“20 bildet den unmittelbaren Anfang der logischen Philosophie, und seine Vermittlung besteht darin, das Ergebnis des Gangs des Bewusstseins in der Phänomenologie des Geistes zu sein. Die Wissenschaft der Logik setzt also die Entwicklung des „reinen Wissens“ der Phänomenologie des Geistes $^{21}$ voraus („Die Logik hat insofern die Wissenschaft des erscheinenden Geistes zu ihrer Voraussetzung“ [GW 21, S. 54]). Jedes ihrer Elemente ist in dem Sinne notwendig vermittelt, als es den Denkraum einer Wissensstruktur voraussetzt, die erst möglich macht, jeden der einzelnen Begriffe - auch den Anfang - in seiner wahren Konzeption zu entfalten. Indem die Logik die absolute Form des Wissens voraussetzt, ist in jedem ihrer Begriffe dessen grundsätzliche Vermittlungsbewegung implizit eingetragen. Erst durch sie nämlich wird die Bewegung der Logik dahingehend möglich, dass eine lokale und eine globale Perspektive auf die jeweilige Kategorie in eine logische Kommunikation miteinander treten, indem die jeweilige begrenzte Selbstbeschreibung der Kategorie in einen bedeutungsgenerierenden Zusammenhang mit dem Wissen über ihre wahren Inhalte, Gründe und Konsequenzen tritt: „Es ist hiemit als factisch falsch aufgezeigt

20 „Die Logik ist die reine Wissenschaft, d.i. das reine Wissen in dem ganzen Umfange seiner Entwicklung. Diese Idee aber hat sich in jenem Resultate dahin bestimmt, die zur Wahrheit gewordene Gewißheit zu seyn [...], die nach der einen Seite dem Gegenstande nicht mehr gegenüber ist, sondern ihn innerlich gemacht hat, ihn als sich selbst weiß, - und die auf der andern Seite das Wissen von sich als von einem, das dem Gegenständlichen gegenüber und nur dessen Vernichtung sey, aufgegeben [hat], dieser Subjectivität entäußert und Einheit mit seiner Entäußerung ist.“ (GW 21, S. 55)

21 So wendet sich die Phänomenologie des Geistes explizit gegen die „natürliche Vorstellung, daß, eh in der Philosophie an die Sache selbst, nemlich an das wirkliche Erkennen dessen, was in Wahrheit ist, gegangen wird, es nothwendig sey, vorher über das Erkennen sich zu verständigen, das als das Werkzeug [...] betrachtet wird.“ (GW 9, S. 53) Damit wird aber das Wahre nicht in seinem An-sich gegeben, sondern zeigt sich gewissermaßen nur durch ein Medium. Für Hegel scheint also der Gebrauch einer Methodologie, die nicht die Form der Sache selbst ist, sondern von außen an sie herangetragen wird, für die Philosophie widersinnig. 
worden, daß es ein unmittelbares Wissen gebe, ein Wissen welches ohne Vermittlung es sey mit Anderem oder in ihm selbst mit sich sey." $\left(\right.$ GW 20, S. 115, § 75) ${ }^{22}$

\begin{abstract}
Was aber ist das ,absolute Wissen', zu dem uns die Phänomenologie führt? Keine besondere Form eines jenseits des normalen Wissens existierenden ,mystischen` oder gewöhnlichen Sinne ,spekulativen' Wissens. Vielmehr [...] ein System, das darauf beruht, daß es keine unüberwindbaren ontologischen Gegensätze zwischen Geist und Natur, Materie und Denken, Individuum und Kultur, Einzelnem und Allgemeinem gibt. Diese Begriffe und Gegenstände lassen sich vielmehr als stufenweise Selbstdifferenzierung und Selbstreflexion einer einzigen geistigen Wirklichkeit erklären. [...] Dieses Transparent-Werden von Wirklichkeit und Selbst - als im Grunde Identischen - ist das ,absolute Wissen. ${ }^{23}$
\end{abstract}

Das reine oder absolute Wissen ist das Bewusstsein, welches begriffen hat, dass der Gegensatz von Denken und Gegenstand, von Subjekt und Objekt eine letztgültige Einheit bildet. ${ }^{24}$ Die Phänomenologie zeichnet den Weg nach, in dessen Verlauf das natürliche Bewusstsein über seinen Anfang, den vielfältigen Graben zwischen Bewusstsein und Wirklichkeit, wie er sich vom reinen Wissen aus darstellt, hinauskommt. ${ }^{25}$ Die Aufgabe der Phänomenologie besteht demnach darin, in der Entwicklungsgeschichte des Geistes zu zeigen, inwiefern diese Unmittelbarkeit nur ein unentwickeltes bzw. unterentwickeltes Bewusstsein darstellt, „dessen Sein und Wahrheit erst über die stufenweise Entwicklung expliziert wird“. ${ }^{26}$ Indem der Anfang der Logik auf dem Vermittlungsergebnis der Phänomenologie aufbaut, hebt er seine absolute Unmittelbarkeit also in globaler Systemperspektive immer schon auf. In der globalen Logik der Wahrheit, wie sie sich in Hegels Gesamtsystem seiner Schriften zeigen soll, sind die Logik und damit ihre

22 Damit werden Positionen problematisch, welche die Phänomenologie des Geistes überhaupt nicht als wesentlich für Hegels entwickelte Logik annehmen. Hegel führt hier nämlich selbst vor, inwiefern deren Ergebnisse und spezifischer Argumentationsverlauf mehr als nur „Propädeutik“ seines Systems sind.

23 Ludwig Siep: Der Weg der Phänomenologie des Geistes. Ein einführender Kommentar zu Hegels „Differenzschrift“ und „Phänomenologie des Geistes“, Frankfurt am Main 2000, S. 255 f. Zur begrifflichen Differenz zwischen „absolutem“ und „reinem“ Wissen vgl. Lu De Vos (,Wissen, reines.“, in: Hegel-Lexikon, hg.v. Paul Cobbe, Darmstadt 2006, S. 502-507, hier: S. 504): „Mit dem Prädikat ,rein', das das Prädikat ,absolut' ersetzt, ändert sich die logische Bedeutung nicht.“ 24 „Das reine Wissen ist [...] Wissen ohne Gegensatz [...] und [...] dennoch nicht leer.“ (De Vos: „Wissen, reines“, S. 504).

25 Vgl. GW 21, S. 35: „Aber die Befreyung von dem Gegensatze des Bewußtseyns, welche die Wissenschaft muß voraussetzen können, erhebt die Denkbestimmungen über diesen ängstlichen, unvollendeten Standpunkt und fordert die Betrachtung derselben, wie sie an und für sich, ohne eine solche Beschränkung und Rüksicht, das Logische, das Rein-vernünftige sind.“

26 Emil Angehrn: Die Frage nach dem Ursprung. Philosophie zwischen Ursprungsdenken und Ursprungskritik, München 2007, S. 171. 
scheinbar unmittelbaren Anfangskategorien (Sein und Nichts) bereits vermittelte Endprodukte.

Es ist nicht von der Hand zu weisen, dass die Vollständigkeit für sich selbst als Anspruch des Begründungs- und Entwicklungsprogramms der Logik unbedingt ernst genommen werden muss: Die Logik beansprucht, nichts anderes vorauszusetzen als die Möglichkeit des reinen Entschlusses, überhaupt zu denken ${ }^{27}$. Indem man aber auf die Voraussetzung der Phänomenologie des Geistes verweist, wird nur allzu oft dieses Programm als Ganzes verabschiedet und so auch die Rechtfertigung der Möglichkeit vergessener oder verschwiegener Voraussetzungen - mit Hinweis auf diese Öffnung - undiskutiert eingekauft. Wie erneut Vittorio Hösle aber zu Bedenken gegeben hat, ist die Beziehung auf die Phänomenologie, rein von der Logik und ihrem Systemanspruch aus gesehen, nur „,von psychologischer, nicht geltungstheoretischer Bedeutung“, weil „der Begriff der Wissenschaft als jener Erkenntnisform, die den Gegensatz des Bewußtseins überwunden hat, ,innerhalb der Logik selbst hervorgeht', die als sich selbst begründendes und insofern voraussetzungsloses System gedeutet werden muß.“28 Der Verweis auf die Phänomenologie hilft also demnach weder dabei, Hegels Projekt einer sich vollständig selbst begründenden Logik als von vornherein gescheitert $\mathrm{zu}$ betrachten, noch erlaubt er es, unter Verweis auf notwendige äußere Voraussetzungen wie die Phänomenologie auch bestimmte Aspekte der Methode aus dem Gang der Kategorienentwicklung und damit ihrer inneren Begründung im Raum der Logik auszugliedern. M. E. gilt es hier, beide sich scheinbar ausschließenden Perspektiven auf die Phänomenologie als Aspektverdopplung zu verstehen, die mit verschiedenen Brennweiten der Betrachtung von Hegels System gekoppelt ist. Natürlich fügt sich die Logik einerseits in das Gesamtsystem der hegelschen Philosophie ein, wenn ihr Systemanspruch ernst genommen und zugleich mit dem Systemanspruch des Ganzen vermittelt wird: Dann aber muss sie notwendig auch so gedacht werden, dass sie an Resultate anderer Systemteile anschließt und diese aufgreift. Andererseits aber sind diese Resultate mit Blick auf die Logik

27 Vgl. zum Entschluss, rein denken zu wollen, als Anfang der Logik Klaus Vieweg: „Der Anfang der Philosophie. Hegels Aufhebung des Pyrrhonismus“, in: Wolfgang Welsch, Klaus Vieweg: Das Interesse des Denkens. Hegel aus heutiger Sicht, München 2003, S. 131-146. Stephen Houlgate hat zurecht darauf verwiesen, dass ,the decision made by the thinker to focus on pure being does not constitute a founding presupposition of the Logic: one that determines in advance the path that the Logic will take“ (Houlgate: The Opening of Hegel's Logic, S. 90). Und weiter: „The decision to think pure being at the start of the Logic initiates a study of the basic categories of thought rather than action in the world. Nevertheless, as a decision or act of will, it is the same as the act described in the Philosophy of Right: an act of abstraction by thought." (Houlgate: The Opening of Hegel's Logic, S. 90)

28 Hösle: Hegels System, S. 66; in gleicher Weise Iber: Metaphysik absoluter Relationalität, S. 10. 
selbst nur als solche Voraussetzungen zu behandeln, die erst dann angenommen werden können, wenn sie sich auch innerhalb der Logik zureichend entwickeln, darstellen und begründen lassen, und die unabhängig davon - d. h. als bloße, in der Logik nicht auf eigensinnige (logische) Weise einholbare und wiederholbare Voraussetzungen - in der Tat keine geltungstheoretische Bedeutung für die Logik haben können. Schließlich ist die Logik der Voraussetzung selbst ein tragender Pfeiler der Wesenslogik: und in ihr das Angemessenheitskriterium alles Voraussetzens, niemals bloßes Vorausliegendes zu sein. Es gilt also, das Verhältnis der Logik zur Phänomenologie des Geistes in der Komplexität zu denken, die durch die Systemstruktur bei Hegel als „Kreis von Kreisen“ (GW 12, S. 252) nahelegt wird.

Anton Friedrich Koch und Wolfgang Welsch haben auf die evolutionäre Kraft der Kategorien verweisen, die wirklich neue Strukturen erzeugen und keine „fertige[n], statische[n], ewige[n] Gegebenheit[en] “29 darstellen, welche einer bloßen „Entwicklungslogik“30 folgen. Was die Welt bewegt, als das „We s e n tli c he, das In nere, das Wahre “ (GW 20, S. 66, § 21) der Wirklichkeit, sind die sich in ihr verwirklichenden logischen Strukturen, als das Programm des Realen, wie sie die Wissenschaft der Logik vor aller Realphilosophie als Ganzes zu geben beansprucht. Klaus Vieweg hat an diese Hegelianische Einsicht anschließend in einer genauen und umfassenden Detailanalyse gezeigt, in welcher Weise dies im Besonderen für den Bereich der Rechtsphilosophie und damit für die Gestalt des objektiven Geistes gilt und welche grundsätzlichen Perspektiven und Gehalte erst sichtbar werden, wenn man die enge Interaktion zwischen dem Bereich des Logischen und der historischen Welt sozialer Institutionen in den Blick nimmt. ${ }^{31}$ Diese logische „Vorprogrammierung“ allerdings, wenn man so will, ist in der Forschung nicht frei von Zweifel geblieben. In der Kritik an Hegels absoluter logischen Fundierung des Realen hat Wofgang Welsch deshalb die sehr hilfreiche begriffliche Unterscheidung zwischen „Evolution“ und „Entwicklung“ bei Hegel eingeführt. Einerseits ist nach Welsch der logische Prozess „selbstgenerativ“, d. h. er hat den Charakter der „Selbsthervorbringung“323; „die logische Bewegung ist nicht die Ausfaltung eines anfänglichen Keims, in dem das Programm des Fol-

29 Anton Friedrich Koch: Die Evolution des logischen Raumes. Aufsätze zu Hegels NichtstandardMetaphysik, Tübingen 2014, S. 4.

30 Vgl. Welsch: Absoluter Idealismus und Evolutionsdenken, S. 658-661.

31 Vgl. Klaus Vieweg: Das Denken der Freiheit. Hegels Grundlinien der Philosophie des Rechts, München 2012. Auch der Band Hegel's Political Philosophy. On the Normative Significance of Method and System (hg.v. Thom Brooks/Sebastian Stein, Oxford 2017) darf hier, wenn es um den Zusammenhang von Logik und Rechtsphilosophie geht, als einschlägig gelten.

32 Welsch: Absoluter Idealismus und Evolutionsdenken, S. 657. 
genden schon bereitläge. Sondern die logische Bewegung erzeugt genuin all das, was in diesem Verlauf auftritt und nur durch diesen Prozeß entstehen kann“"33. Damit kommt ihr der Charakter der „Evolution“ zu, wohingegen die Realphilosophie bzw. das Wirkliche selbst nur als „Entwicklung“ zu fassen ist: „Entwicklung bezeichnet einen Vorgang, in dem etwas, das an sich schon vorhanden ist, zur Entfaltung gelangt.““34 Oder mit Hegel: Der Begriff der „Entwicklung“ bezeichnet „eine innere Bestimmung, eine an sich vorhandene Voraussetzung“ (TWA 12, S. 75), die schon zugrunde liegt und welche sich dann nur noch „zur Existenz“ (TWA 12, S. 75) bringt. Folglich konstatiert Welsch: „Das Grundbild ist also dies, daß die logische Bewegung den Status einer originären Evolution besitzt, das Realgeschehen hingegen, weil ihm die logischen Formen zugrunde liegen, nur noch den Status einer Entwicklung haben kann. Das Realgeschehen kann nur noch entfalten, was dem Grundriß nach logisch schon vorgezeichnet ist." ${ }^{\text {"35 }}$ Welschs Korrekturvorschlag zielt deshalb darauf, logische mit realen Strukturen gleichursprünglich zu denken. Sichtbar werden soll dadurch, dass die Frage nach der Voraussetzungslosigkeit in der Logik bzw. der Logik als solcher wesentliche Konsequenzen für die Realphilosophie nach sich zieht, wo bestimmte Potenziale und Dimensionen von Freiheit durch den fundierenden Charakter der Logik in Hegels System bedroht zu sein scheinen, wenn man „die Evolution des logischen Raumes“ (Koch) einzig einer nachträglichen „Entwicklung“ vorschaltet.

33 Welsch: Absoluter Idealismus und Evolutionsdenken, S. 659.

34 Welsch: Absoluter Idealismus und Evolutionsdenken, S. 658.

35 Welsch: Absoluter Idealismus und Evolutionsdenken, S. 664. 